\title{
Homeomorphisms of fractafolds
}

\author{
by \\ Ying Ying Chan (Hong Kong) and \\ Robert S. Strichartz (Ithaca, NY)
}

\begin{abstract}
We classify all homeomorphisms of the double cover of the Sierpiński gasket in $n$ dimensions. We show that there is a unique homeomorphism mapping any cell to any other cell with prescribed mapping of boundary points, and any homeomorphism is either a permutation of a finite number of topological cells or a mapping of infinite order with one or two fixed points. In contrast we show that any compact fractafold based on the level-3 Sierpiński gasket is topologically rigid.
\end{abstract}

1. Introduction. Bandt and Retta $[\mathrm{BR}]$ showed that the Sierpiński gasket and related fractals are topologically rigid: any homeomorphism of the space onto itself must be an isometry. This is a striking property that appears to be unique to the fractal world.

Recall that the Sierpiński gasket, which we will denote by $S G^{2}$, is the unique non-empty compact subset $K$ of the hyperplane $\left\{x_{1}+x_{2}+x_{3}=1\right\}$ in $\mathbb{R}^{3}$ satisfying

$$
K=\bigcup_{i} F_{i} K
$$

where

$$
F_{i} x=\frac{1}{2}\left(x+e_{i}\right)
$$

and $e_{i}$ are the standard basis vectors in $\mathbb{R}^{3}$. We call $\left\{F_{1}, F_{2}, F_{3}\right\}$ the iterated function system (IFS) that generates $S G^{2}$, and we call $S G^{2}$ the attractor of the IFS. See Figure1. Related fractals may be generated by different choices of IFS. For example, the $n$-dimensional Sierpiński gasket $S G^{n}$ is the subset of the hyperplane $\left\{\sum_{i=1}^{n+1} x_{i}=1\right\}$ defined by (1) and (2) for $i=1, \ldots, n+1$. A different 2-dimensional fractal is the level-3 Sierpiński gasket $S G_{3}$ defined by a 6 -element IFS; see Figure 2 and Section 3 for a precise description. 


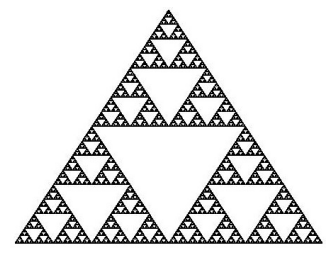

Fig. 1. $S G^{2}$

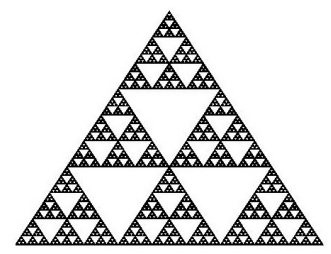

Fig. 2. $S G_{3}$

However, these fractals are analogs of the unit interval. Better fractal analogs of closed manifolds are provided by the class of fractafolds introduced by the second author [S1]. Roughly speaking, given a fractal $K$, a fractafold $\mathcal{F}$ based on $K$ is a topological space where every point has a neighborhood homeomorphic to an open set in $K$. The fractals we consider belong to the class of PCF self-similar sets defined by Kigami [Ki], and come with a finite set of boundary points. (In the case of the $n$-dimensional Sierpiński gasket $S G^{n}$ the boundary consists of the $n+1$ vertices of an $n$-simplex.) A compact fractafold $\mathcal{F}$ consists of a finite number of copies of $K$ with some boundary points identified. If all boundary points are identified, then $\mathcal{F}$ is a compact fractafold without boundary (otherwise, the boundary of $\mathcal{F}$ is the set of boundary points of the copies of $K$ that are not identified).

The simplest example is the double cover of $K$, where two copies of $K$ have all boundary points pairwise identified. This is analogous to the circle, which is obtained by identifying the boundary points of two copies of the unit interval. It was already pointed out in [S1] that the double cover of $S G^{2}$ is not topologically rigid. In fact there is a homeomorphism of infinite order that is expanding in some cells and contracting in other cells, where we call a subset of $K$ a cell if it is the image of $K$ under compositions of mappings in the IFS. More generally, a topological cell is any subset of $K$ homeomorphic to $K$.

The first goal of this paper is to give a complete description of all homeomorphisms of the double cover of $S G^{n}$. One of our main results is that given any two topological cells in $\mathcal{F}$, there exists a unique homeomorphism of $\mathcal{F}$ mapping one to the other, with the mapping prescribed on the boundary. As a consequence we show that there are two types of homeomorphisms $h$ : 1) $h$ is a permutation of a finite number of topological cells in a certain decomposition of $\mathcal{F}$, hence $h$ has finite order; 2) $h$ has infinite order with one or two fixed points. In the first case the decomposition of $\mathcal{F}$ is either a set of two topological cells, so $h$ belongs to a group of homeomorphisms isomorphic to $S_{n} \times \mathbb{Z}_{2}$, or the decomposition consists of $n+1$ topological cells, so $h$ belongs to $S_{n+1}$, where $S_{n}$ denotes the permutation group on $n$ letters. In the second case, if $h$ has one fixed point then that point is a 
junction point (a point where two cells intersect), and $h$ is expanding on one side of the junction point and contracting on the other side; if $h$ has two fixed points then one determines the other, and they are both eventually periodic points.

It is natural to ask what are the homeomorphisms of more general compact fractafolds based on $S G^{n}$. We will see that the answer is rather uninteresting. Although there are homeomorphisms of infinite order on some other fractafolds, these homeomorphisms essentially commute and have no interesting interactions.

The second main goal of this paper is to show that for $K=S G_{3}$, every compact fractafold is topologically rigid. The space $S G_{3}$ shares the property with $S G^{n}$ that the boundary points are topologically different from all other points, so that a compact fractafold again consists of an infinite number of copies of $K$ with some boundary points identified. Here we may have two or three boundary points identified, as there are junction points in $K$ where two or three cells come together. Why is the case of $S G_{3}$ so different from $S G^{n}$ ? The answer can be understood using the notion of minimal decomposition of a fractafold of $\mathcal{F}$. Of course $\mathcal{F}$ is a finite union of copies of $K$, with certain boundary points identified, but there are many such representations, as each copy of $K$ may be split into cells homeomorphic to $K$. In the other direction, we may be able to combine several copies of $K$ into a set that is homeomorphic to $K$; we say that the decomposition is minimal if this is not possible. Clearly, homeomorphisms of $\mathcal{F}$ map minimal decompositions into minimal decompositions. In the case of $S G_{3}$ we will show that there is a unique minimal decomposition (Theorem 3.5), so this allows us to transfer the rigidity from $S G_{3}$ to $\mathcal{F}$. In the case $K=S G^{n}$ and $\mathcal{F}$ is the double cover, there are infinitely many minimal decompositions (any cell in either copy and its complement, for example). We conjecture that the rigidity result for $S G_{3}$ extends to all higher level Sierpiński gaskets in $n$ dimensions. For other fractals the situation is more complicated. For example, for the pentagasket

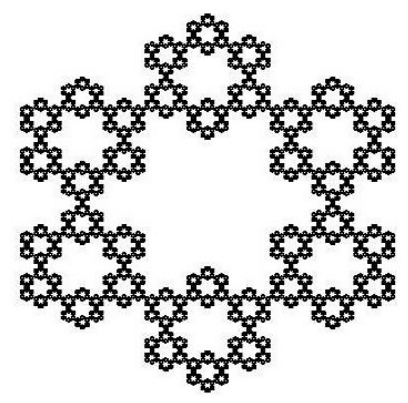

Fig. 3. A hexagasket 
or hexagasket (Figure 3), one can construct a fractafold from two copies by identifying two pairs of boundary points (Figure 4 ) having an infinite family of homeomorphisms, but the double cover (with three identified pairs) is topologically rigid.

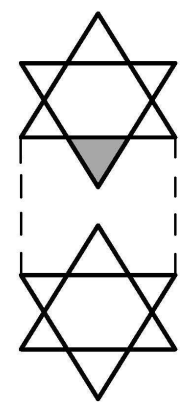

Fig. 4. A schematic diagram of a fractafold constructed from the hexagasket with two identified pairs of points as indicated. The shaded cell and its complement give a minimal decomposition different from the obvious one, leading to an infinite family of homeomorphisms of this fractafold based on the hexagasket.

The fractafolds considered here have been studied intensively from the analytic point of view (see [Ki], [S2], and the references there). Topological properties related to covering maps have been studied in [S3], [RS].

2. The double cover of the Sierpiński gasket. In this section we describe all homeomorphisms of the double cover of $S G^{n}$. We take two copies of $S G^{n}$, which we denote $A$ and $B$. To be specific, we take $A=K$ defined by (1) and (2), while for $B$ we replace the IFS $\left\{F_{i}\right\}$ by $\left\{G_{i}\right\}$ defined by

$$
G_{i} x=\frac{1}{2}\left(x_{i}-e_{i}\right),
$$

so $B$ is contained in the hyperplane $\left\{\sum x_{i}=-1\right\}$.

Definition 2.1. Let $\mathcal{F}=A \cup B / \sim$ where $e_{i} \sim-e_{i}$ for $i=1, \ldots, n+1$. We call $\mathcal{F}$ the double cover of the Sierpinski gasket and let $\varphi: A \cup B \rightarrow(A \cup B) / \sim$ be the corresponding quotient map.

A word $w$ of length $m=|w|$ is defined to be a sequence $w=\left(w_{1}, \ldots, w_{m}\right)$ where each $w_{j}$ is chosen from $\{1, \ldots, n+1\}$. We write $F_{w}=F_{w_{1}} \circ \cdots \circ F_{w_{m}}$. By iterating (1) we obtain

$$
A=\bigcup_{|w|=m} F_{w} A \text { and } B=\bigcup_{|w|=m} G_{w} B .
$$

We write $A_{w}=F_{w} A$ and $B_{w}=G_{w} B$, and refer to these as cells of order $m$. The boundary of the cell $F_{w} A$ is the set $\left\{F_{w} e_{i} \mid i=1, \ldots, n+1\right\}$ and similarly for $F_{w} B$ with $e_{i}$ replaced by $-e_{i}$. We can also use infinite 
words $w=\left(w_{1}, w_{2}, \ldots\right)$ to parametrize points, and write $x=A_{w}$ for $x=$ $\bigcap_{k=1}^{\infty} A_{\left(w_{1}, \ldots w_{k}\right)}$. Note that the boundary points $e_{i}$ correspond to the words $\bar{i}=(i, i, \ldots)$. There is a similar description for points in $B$. Points that lie on the boundary of a cell, and hence in two cells, have two different parametrizations, and are called junction points.

One of the main results of $\left[\mathrm{BR}\right.$ is that any homeomorphism of $S G^{n}$ is given by

$$
h\left(x_{1}, \ldots, x_{n+1}\right)=\left(x_{t(1)}, \ldots, x_{t(n+1)}\right)
$$

for some $t \in S_{n+1}$. In this section we give a complete description of all homeomorphisms of $\mathcal{F}$. The main result (Theorem 2.8) is that there is a unique homeomorphism mapping one topological cell to another with specified permutation of the boundaries. A topological cell is a subset of $\mathcal{F}$ homeomorphic to $S G^{n}$. We will show that the closure of the complement of a cell is a topological cell, and together with the cells these are the only possibilities. We then show that there are two different types of homeomorphisms depending on whether they generate infinite or finite subgroups.

We begin by describing a set of homeomorphisms that generate the group of all homeomorphisms of $\mathcal{F}$.

(i) For all permutations $t \in S_{n+1}$, define a homeomorphism $f_{t}$ on each of $A$ and $B$ by $f_{t}\left(x_{1}, \ldots, x_{n+1}\right)=\left(x_{t(1)}, \ldots, x_{t(n+1)}\right)$. Then $f_{i}$ maps $A_{i}$ one-to-one onto $A_{t(i)}$ and $B_{i}$ one-to-one onto $B_{t(i)}$, for all $i \in$ $\{1, \ldots, n+1\}$, and $\varphi \circ f_{i} \circ \varphi^{-1}$ gives a homeomorphism on $\mathcal{F}$.

(ii) $\mu(x)=-x$ also defines a homeomorphism $\varphi \circ \mu \circ \varphi^{-1}$ on $\mathcal{F}$ that maps $A_{w}$ onto $B_{w}$.

(iii) Another homeomorphism of $\mathcal{F}$ is given by $\varphi \circ h_{1} \circ \varphi^{-1}$ where

$$
h_{1}(x)= \begin{cases}F_{1}^{-1}(x) & \text { if } x \in A_{1}, \\ G_{i}\left(-f_{(1, i)}\left(F_{i}^{-1}(x)\right)\right) & \text { if } x \in A_{i}, i \in\{2, \ldots, n+1\}, \\ G_{1}(x) & \text { if } x \in B .\end{cases}
$$

It maps $A_{1}$ onto $A, A_{i}$ onto $B_{i}$ for $i \neq 1$, and $B$ onto $B_{1}$ with $A_{\overline{1}}=$ $B_{\overline{1}}$ as fixed point. It is possible to realize this homeomorphism as a Möbius transformation on the disk with respect to the Apollonian realization of $\mathcal{F}$. See Chapter 7 of [MSW] for beautiful illustrations of this realization. More generally, $\varphi \circ h_{i} \circ \varphi^{-1}$ where $h_{i}=f_{(1, i)} \circ h_{1} \circ$ $f_{(1, i)}$ maps $A_{i}$ onto $A$ with $A_{\bar{i}}=B_{\bar{i}}$ as fixed point. We may write $h_{j}(x)= \begin{cases}F_{j}^{-1}(x) & \text { if } x \in A_{j}, \\ G_{i}\left(-f_{(j, i)}\left(F_{i}^{-1}(x)\right)\right) & \text { if } x \in A_{i}, i \in\{1, \ldots, n+1\} \backslash\{j\}, \\ G_{j}(x) & \text { if } x \in B .\end{cases}$ For any word $w=\left(w_{1}, \ldots, w_{m}\right)$ we write $h_{w}=h_{w_{m}} \circ \cdots \circ h_{w_{1}}$. 
Consider a cell $A_{w}$ where $w=\left(w_{1}, \ldots, w_{m}\right)$ and $t \in S_{n+1}$. Note that $h_{w}\left(A_{w}\right)=h_{w_{m}} \circ \cdots \circ h_{w_{1}}\left(A_{w}\right)=F_{w_{m}}^{-1} \circ \cdots \circ F_{w_{1}}^{-1} \circ F_{w_{1}} \circ \cdots \circ F_{w_{m}}(A)=A$ and $f_{t} \circ h_{w}\left(A_{w, \bar{i}}\right)=A_{\overline{t(i)}}$. It follows from [BR] that $\varphi \circ f_{t} \circ h_{w} \circ \varphi$ is the unique homeomorphism that maps $A_{w}$ onto $A$ and $A_{w, \bar{i}}$ to $A_{\overline{t(i)}}$. Indeed, if there exist two homeomorphisms $f_{1}$ and $f_{2}$ with such properties, then $f_{2} \circ f_{1}^{-1}$ is a homeomorphism that maps $B$ onto $B$ and $A$ onto $A$ with $B_{\bar{i}}$, $i=1, \ldots, n+1$, as fixed points. Since both $B$ and $A$ are $S G^{n}$, this implies that $f_{1}=f_{2}$.

Given a cell $B_{w}$ where $w=\left(w_{1}, \ldots, w_{m}\right)$, we have $h_{w} \circ \mu\left(B_{w}\right)=h_{w}\left(A_{w}\right)$ $=A$. This means that given any two cells $C_{1}$ and $C_{2}$ with orderings of the boundary points, in $A$ or $B$, there is a unique homeomorphism that maps $C_{1}$ onto $C_{2}$ and preserves the ordering of the boundary points.

Definition 2.2. Let $S^{*}=\bigcup_{k=1}^{\infty}\{1, \ldots, n+1\}^{k}$. A set $C$ is called a topological cell if it is homeomorphic to $A$, and is called a cell if there exists $w \in S^{*}$ such that $C=A_{w}$ or $C=B_{w}$. Clearly, all cells are topological cells.

Definition 2.3. Let $G$ be a topological cell. It is shown in $[\mathrm{BR}]$ that $G$ can be decomposed into $n+1$ topological cells in a unique way. We call the $n+1$ topological cells 1 -cells of $G$.

The following lemma is an immediate consequence.

Lemma 2.4. Let $h$ be a homeomorphism on $\mathcal{F}$. If $G_{1}$ is a 1-cell of $G$, then $h\left(G_{1}\right)$ is a 1-cell of $h(G)$.

Following [BR], $x \in A$ is called a cutpoint of $A$ if $A \backslash\{x\}$ is disconnected, and it is called a local cutpoint of $A$ if $U \backslash\{x\}$ is not connected for some connected neighborhood $U$ of $x$. Moreover, if $x$ is a cutpoint or local cutpoint of $A$ then $x$ must be of the form $f_{w}\left(e_{i}\right)$ for some $w \in \Omega^{*}$ and some boundary point $e_{i}$. Similarly, we define cutpoints and local cutpoints of topological cells.

Lemma 2.5. Let $C$ be a topological cell. A boundary point of $C$ cannot be a local cutpoint of $C$.

Proof. Let $h_{C}$ be a homeomorphism from $A$ onto $C$. We can then use $h_{C}$ to transfer the result from $A$ to $C$.

Lemma 2.6. If $C$ and $D$ are two topological cells with disjoint interiors, then one of them must be a cell.

Proof. It is shown in $[\mathrm{BR}$ that any topological cell contained in $A$ (or $B$ ) must be a cell so it suffices to show that it is impossible for both $C$ and $D$ to have nonempty intersection with the interiors of both $A$ and $B$. If this was the case, then removing all the common boundary points $\left\{e_{i}\right\}$ in the interior of $C$ would disconnect $C$ and similarly for $D$. Thus, either $C$ or $D$ could be 
disconnected by removing at most $[(n+1) / 2]$ points, and this contradicts Theorem 4.1 of $[\mathrm{BR}]$.

Given a set $X \subset \mathcal{F}$, denote the closure of the complement of $X$ in $\mathcal{F}$ by $X^{c}$.

Lemma 2.7. Let $D$ be a topological cell. Then $D^{c}$ is also a topological cell, and either $D$ or $D^{c}$ is a cell.

Proof. There exists a cell $E$ in $\mathcal{F}$ such that $D$ and $E$ are disjoint, since $D$ is compact and $D=\mathcal{F}$ is clearly not possible. Thus, there exists a homeomorphism $f_{1}$ such that $f_{1}(E)=A$. This implies that $f_{1}(D) \subseteq B$. By [BR, since $f_{1}(D)$ is homeomorphic to $S G^{n}$, it must be a cell in $B$. Let $f_{2}$ be a homeomorphism such that $f_{2}\left(f_{1}(D)\right)=A$. Then $D^{c}=f_{1}^{-1}\left(f_{2}^{-1}(B)\right)$ is homeomorphic to $S G^{n}$. It follows from Lemma 2.6 that either $D$ or $D^{c}$ is a cell.

TheOREM 2.8. Given two topological cells $C$ and $D$ with boundary points $\left\{x_{1}, \ldots, x_{n+1}\right\}$ and $\left\{y_{1}, \ldots, y_{n+1}\right\}$ respectively. Let $t \in S_{n+1}$. There exists a unique homeomorphism $f$ on $\mathcal{F}$ such that $f(C)=D$ and $f\left(x_{i}\right)=y_{t(i)}$ for all $i \in\{1, \ldots, n+1\}$.

Proof. By Lemma 2.7, either $D$ or $D^{c}$ is a cell. By the construction in (iii), there is a homeomorphism $f_{1}$ that maps $D$ onto $A$ and $D^{c}$ onto $B$, with any prescribed behavior on boundary points. Similarly, there is a homeomorphism $f_{2}$ that maps $C$ onto $A$ and $C^{c}$ onto $B$. Then $f_{1}^{-1} \circ f_{2}$ is the desired homeomorphism. The uniqueness again follows by the rigidity of $S G^{n}$.

Lemma 2.9. Given a topological cell $T$ in $\mathcal{F}$, there is a unique decomposition of $\mathcal{F}$ into $n+2$ topological cells where $T$ is one of the cells. Denote the set of the $n+2$ topological cells by $\Gamma_{T}$. If $h$ is a homeomorphism on $\mathcal{F}$ that maps a topological cell $C$ onto a topological cell $D$, then $h$ maps $\Gamma_{C}$ to $\Gamma_{D}$.

Proof. We take $\Gamma_{T}$ to be $T$ together with the 1-cells of $T^{c}$.

Lemma 2.10. Given a topological cell $G$ and a topological cell $E \subsetneq G$, there exist $n$ other topological cells $E_{2}, \ldots, E_{n+1} \in \Gamma_{E}$ such that $E_{2} \cup \cdots \cup$ $E_{n+1} \cup E$ is a topological cell in $G$.

Proof. We may assume that $G=A$. Then $E=A_{w}$ for some $w=$ $\left(w_{1}, \ldots, w_{m}\right)$. If $m=1$ the result is trivial. If $m>1$, then $\Gamma_{E}$ is the union of $A_{\left(w_{1}, \ldots, w_{m-1}, i\right)}$ for $1 \leq i \leq n+1$ and $A_{\left(w_{1}, \ldots, w_{m-1}\right)}^{c}$. Clearly, the union of all elements in $\Gamma_{E}$ except $A_{\left(w_{1}, \ldots, w_{m-1}\right)}^{c}$ is a cell in $G$.

Next we distinguish two types of homeomorphisms of $\mathcal{F}$ :

Type 1: Homeomorphisms $h$ such that there exists a topological cell $C$ with $h(C) \subsetneq C$ or $h(C) \supsetneq C$. These homeomorphisms have infinite order. 
Type 2: Homeomorphisms of finite order which permute either $n+2$ topological cells in some $\Gamma_{C}$, or which permute the two topological cells $C$ and $C^{c}$.

Let us first consider a homeomorphism such that $h(A) \subsetneq A$. We have $A \supsetneq h(A) \supsetneq h^{2}(A) \supsetneq h^{3}(A) \supsetneq \cdots$. Clearly, $h$ is of infinite order.

LEMma 2.11. $\bigcap_{k=0}^{\infty} h^{k}(A)$ and $\bigcap_{k=0}^{\infty}\left(h^{-1}\right)^{k}(B)$ are fixed points of $h$ and there are no other fixed points.

Proof. $h$ restricted to $A$ is a contractive similarity, and $h^{-1}$ restricted to $B$ is also a contractive similarity. It follows that $\bigcap_{k=0}^{\infty} h^{k}(A)$ is a singleton and a fixed point of $h$, and similar for $\bigcap_{k=0}^{\infty}\left(h^{-1}\right)^{k}(B)$.

If there were another fixed point $z$, either $z \in A$ or $z \in B$. Then either $z \in \bigcap_{k=0}^{\infty} h^{k}(A)$ or $z \in \bigcap_{k=0}^{\infty}\left(h^{-1}\right)^{k}(B)$.

For all $t \in S_{n+1}$ and $w=\left(w_{1}, w_{2}, \ldots\right)$, denote $t(w)=\left(t\left(w_{1}\right), t\left(w_{2}\right), \ldots\right)$. Let $h(A)=A_{w}$ where $w=\left(w_{1}, \ldots, w_{m}\right)$. Then $h=h_{w}^{-1} \circ f_{t}$ for some $t \in S_{n+1}$. In addition, $h^{n}(A)=A_{w, t(w), t^{2}(w), \ldots, t^{n-1}(w)}$ and $\left(h^{-1}\right)^{n}\left(A^{c}\right)=$ $B_{t^{-1}\left(w^{\prime}\right), t^{-2}\left(w^{\prime}\right), \ldots, t^{-n}\left(w^{\prime}\right)}$ where $w^{\prime}=\left(w_{m}, \ldots, w_{1}\right)$. Therefore, the fixed points are

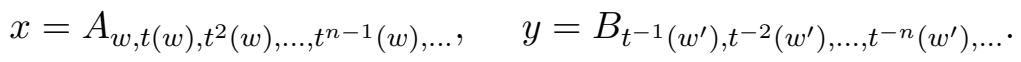

Since $t$ is of order at most $n+1$, we have $t^{d}=$ id for some $d$ satisfying $1 \leq$ $d \leq n+1$. Therefore, $x=A_{\bar{v}}$ and $y=A_{\overline{v^{\prime}}}$, for some $v=\left(v_{1}, \ldots, v_{m}\right) \in \Omega^{*}$ where $v^{\prime}=\left(v_{m}, \ldots, v_{1}\right)$. Moreover, $x=y$ if and only if $x=y=A_{\bar{i}}$ for some $i \in\{1, \ldots, n+1\}$. This occurs if and only if $t$ is the identity map and $w \in\{i\}^{m}$ for some $m \in \mathbb{N}$ and $i \in\{1, \ldots, n+1\}$. In addition, this is the only case that has a fixed point at a boundary point.

Since for all topological cells $C$, there exists a homeomorphism $\phi$ such that $\phi(C)=A$, other homomorphisms of type 1 can be handled similarly by considering $\phi^{-1} \circ h \circ \phi$.

TheOREM 2.12. Any homeomorphism of $\mathcal{F}$ is either of type 1 or of type 2 .

Proof. Suppose $h$ is not of type 1. By Lemma 2.6, either $h(A)$ or $h(B)$ must be a cell. Without loss of generality $h(A)$ is cell, so either $h(A) \subseteq A$ or $h(A) \subseteq B$. Since $h$ is not of type 1 , in the first case we have $h(A)=A$ and $h$ is a trivial permutation of $A, B$ (it is also a permutation of $\Gamma_{A}$ or $\Gamma_{B}$ ) so it is of type 2 .

If $h(A) \subseteq B$ then $h(A)$ is a $k$-cell in $B$ for some $k$. Depending on whether $k$ is even or odd, we will show that $h$ is a permutation of $C$ and $C^{c}$ where $C$ is a $k / 2$-cell in $B$, or $h$ is a permutation of the cells in $\Gamma_{C}$ where $C$ is a $(k+1) / 2$-cell in $B$. This is obvious if $k=0$ when $h(A)=B$ so $h$ permutes 
$A$ and $B$. It is also clear when $k=1$ for then $h(A) \in \Gamma_{A}$ and $h$ permutes $\Gamma_{A}$ by Lemma 2.9 .

More generally, let $B^{\prime}$ denote the $(k-1)$-cell containing $h(A)$. Since $h$ maps the sets in $\Gamma_{A}\left(=A, B_{1}, \ldots, B_{n+1}\right)$ to some permutation of the sets in $\Gamma_{h(A)}$, and since $\left(B^{\prime}\right)^{c} \in \Gamma_{h(A)}$, there must be some value of $j$ such that $h\left(B_{j}\right)=\left(B^{\prime}\right)^{c}$. This means $h\left(A^{\prime}\right)=B^{\prime}$ where $A^{\prime}=A \cup \bigcup_{l \neq j} B_{l}$ is a topological cell containing $A$. Iterating this argument, we obtain a sequence $A \subseteq A^{\prime} \subseteq A^{\prime \prime} \subseteq \cdots \subseteq A^{(m)}$ of topological cells such that $h\left(A^{(m)}\right)=B^{(m)}$ is a $(k-m)$-cell in $B$ and $h(A) \subseteq B^{\prime} \subseteq B^{\prime \prime} \subseteq \cdots \subseteq B^{(m)}$. This process stops when $m=k / 2$ for $k$ even, in case $B^{(m)}=\left(A^{(m)}\right)^{c}$, so $h$ permutes the two, or $m=(k-1) / 2$ when $k$ is odd, in which case $B^{(m)} \in \Gamma_{A^{(m)}}$ and $h$ permutes $\Gamma_{A^{(m)} \text {. }}$.

REMARK. If we fix a topological cell $C$, then there is a unique homeomorphism that realizes any permutation of the cells in $\Gamma_{C}$. Thus we have a finite subgroup of homeomorphisms isomorphic to the symmetric group $S_{n+2}$. If $h(C)=C, h$ permutes the cells in $\Gamma_{C}$, so we are in the previous case. Finally, if $h$ interchanges $C$ and $C^{c}$, then $h$ permutes the $n+1$ boundary points of $C$ (they are also the boundary points of $C^{c}$ ), and any such permutation may be uniquely realized.

We conclude this section with a brief discussion of homeomorphisms of other compact fractafolds based on $S G^{n}$. Suppose $\mathcal{F}$ is a union of $N$ copies $A_{1}, \ldots, A_{N}$ with certain pairs of boundary points identified. We may assume that no pair of boundary points of a single $A_{j}$ are identified, for if this were the case we need only split that $A_{j}$ into its $n+11$-cells. Then the structure $\mathcal{F}$ is described by a cell graph $G$, with one vertex $j$ for each $A_{j}$, and an edge joining $j$ to $k$ if a boundary point of $A_{j}$ is identified with a boundary point of $A_{k}$. We may have more than one edge joining $j$ and $k$ if there are several identifications. The cell graph is not unique, but we may assume that it is minimal in the sense that it does not contain a subgraph of $n+1$ vertices that is complete with no multiple edges. Of course we want to assume that $\mathcal{F}$ is connected, which is equivalent to $G$ being connected. Also, each vertex in $G$ has at most $n+1$ edges, since each $A_{j}$ has $n+1$ boundary points. Note that it is not necessary to specify which boundary points are identified, since all permutations of the boundary points of $S G^{n}$ extend to symmetries of $S G^{n}$.

The cell graph of the double cover of $S G^{n}$ consists of two vertices joined by $n+1$ edges. No other cell graph can contain a pair of vertices joined by $n+1$ edges, because of the connectedness assumption. On the other hand, if the cell graph does not contain a pair of vertices joined by $n$ edges, then it is not difficult to show the minimal decomposition is unique, so $\mathcal{F}$ is topologically rigid: any homeomorphism must be a permutation of the $A_{i}$ cells. (Which permutations correspond to homeomorphisms depends on the 
symmetries of the cell graph.) So it remains to understand the case when the cell graph contains pairs of vertices joined by $n$ edges. Note that there may be more than one such pair, but if so the same vertex cannot belong to more than one pair.

Suppose, to be specific, that $A_{1}$ and $A_{2}$ in $\mathcal{F}$ have exactly $n$ identified vertices, say vertices $2, \ldots, n+1$ of $A_{1}$ identified with vertices $2, \ldots, n+1$ of $A_{2}$. Then there exists a homeomorphism of infinite order analogous to $h_{1}$ that fixes vertex 1 of $A_{1}$ and $A_{2}$, and is the identity on the complement of $A_{1} \cup A_{2}$. This homeomorphism, together with the symmetries of $A_{1} \cup A_{2}$ permuting vertices $2, \ldots, n+1$, generates a subgroup isomorphic to $\mathbb{Z} \times S_{n}$. Moreover, if there are more than one pair of cells with exactly $n$ identified vertices, then the analogous subgroups all commute. In general there may be more homeomorphisms than those generated by these subgroups if the cell graph has any symmetries. One would just have to add a finite number of permutation homeomorphisms to generate the full homeomorphism group.

We conclude from this discussion that, aside from the double cover, there are no other fractafolds based on $S G^{n}$ with an interesting homeomorphism group.

3. Fractafolds based on $S G_{3}$. $S G_{3}$ can be represented as a subset $A$ of the hyperplane $\left\{\left(x_{1}, x_{2}, x_{3}\right) \in \mathbb{R}^{3} \mid x_{1}+x_{2}+x_{3}=1\right\}$ such that $A=$ $f_{1}(A) \cup \cdots \cup f_{6}(A)$ where $f_{i}(x)=\frac{1}{3}\left(x+2 e_{i}\right)$ for

$$
e_{i}= \begin{cases}\text { the } i \text { th unit vector } & \text { if } i \in\{1,2,3\}, \\ \frac{1}{2}\left(e_{1}+e_{2}\right) & \text { if } i=4, \\ \frac{1}{2}\left(e_{2}+e_{3}\right) & \text { if } i=5, \\ \frac{1}{2}\left(e_{3}+e_{1}\right) & \text { if } i=6 .\end{cases}
$$

We call $V=\left\{e_{1}, e_{2}, e_{3}\right\}$ the set of boundary points of $A$. Let $Z=\{1, \ldots, 6\}$ and $\Omega^{*}=\bigcup_{k=1}^{\infty} Z^{k}$. For all $w=\left(w_{1}, \ldots, w_{m}\right) \in \Omega^{*}$, we write $A_{w}=f_{w}(A)=$ $f_{w_{1}} \circ \cdots \circ f_{w_{m}}(A)$. We call $w$ a word of length $|w|=m, A_{w}$ a cell of level $m$ of $A$ and the elements in $f_{w}(V)$ the boundary points of $A_{w}$. Let $C_{1}$ and $C_{2}$ be two cells of $A$ of the same level. They are either disjoint or intersect exactly at one boundary point. Let $Q^{\prime}=\left(\bigcup_{k=1}^{6} f_{i}(V)\right) \backslash\left\{f_{i}\left(e_{i}\right) \mid i \in\{1,2,3\}\right\}$. Every element in $Q^{\prime}$ is the intersection of two or three distinct cells of level 1 .

Let $\Omega=Z^{\infty}$. For each point $x$ in $A$, there exists $w_{1} \in Z$ such that $x \in A_{w_{1}}$. Then there exists $w_{2} \in Z$ such that $x \in A_{\left(w_{1}, w_{2}\right)}$, and so on. Continuing the process, we can find $w=\left(w_{1}, w_{2}, \ldots\right) \in \Omega$ such that $\{x\}=$ $\bigcap_{k=1}^{\infty} A_{\left(w_{1}, \ldots, w_{k}\right)}$, and write $x=A_{w}$. Each element in $\Omega$ represents a unique point in $A$ but some points in $A$ can be represented by more than one point in $\Omega$. We can define an equivalence relation $\sim$ on $\Omega$ such that $w^{1} \sim w^{2}$ if and only if $w^{1}$ and $w^{2}$ represent the same point in $A$. It can be shown 
that $C / \sim$ is a simple finite-to-one invariant factor $[\mathrm{BR}$. Moreover, there is a one-to-one correspondence between the elements in $\Omega^{*}$ and cells in $A$ given by $w \leftrightarrow A_{w}$.

The $G_{m}$ hypergraph of $A$ has the cells $\left\{A_{w} \mid w \in \Omega^{*}\right.$ and $\left.|w|=m\right\}$ as vertices. Vertices $A_{w_{1}}, \ldots, A_{w_{n}}$ with $n \geq 2$ are joined by an edge if and only if $A_{w_{1}} \cap \cdots \cap A_{w_{n}} \neq \emptyset[\mathrm{BR}]$. Note that $A_{4} \cap A_{5} \cap A_{6} \neq \emptyset$. Directly checking all the possibilities, we see that the $G_{1}$ graph is edge-balanced and the $G_{2}$ graph is 2-connected [BR].

From $[\mathrm{BR}]$, it can be shown that $A$ has the following properties:

Property 1. The set of all homeomorphisms from $A$ onto $A$ is $\{f: A \rightarrow$ $\left.A \mid f\left(x_{1}, x_{2}, x_{3}\right)=f\left(x_{t(1)}, x_{t(2)}, x_{t(3)}\right), t \in S_{3}\right\}$ [BR, p. 265, Corollary 5.2].

Property 2. If a subset $D$ of $A$ is homeomorphic to $A$, then $D=A_{w}$ for some $w \in \Omega^{*}[\mathrm{BR}$, p. 264, Theorem 5.1].

Property 3. All local cutpoints of $A$ are of the form $f_{w}(q)$ where $w \in \Omega^{*}$ and $q \in Q^{\prime}$ [BR, p. 261, Proposition 2.1].

Property 4. Let $E$ be a finite subset of local cutpoints of $A$ and for all $v \in \Omega^{*}$, let $E_{v}=E \cap f_{v}\left(Q^{\prime}\right)$. Moreover, let $k$ be the number of components of $A \backslash E$ and for all $v \in \Omega^{*}$, let $k_{v}$ be the number of components of $A_{v} \backslash E_{v}$. Then $k-1=\sum_{E_{v} \neq \emptyset}\left(k_{v}-1\right)$ [BR, p. 263, Formula $\left.(* *)\right]$.

Suppose $E$ is a set of two or three points that can disconnect $A$ but no proper subset of $E$ can disconnect $A$. Elements of $E$ must be of the form $f_{w^{i}}\left(p_{i}\right)$ where $w^{i} \in \Omega^{*}$ and $p_{i} \in Q^{\prime}$. Let $r=\max \left\{\left|w^{i}\right| w^{i} \in \Omega^{*}\right.$ and $\left.f_{w^{i}}\left(Q^{\prime}\right) \cap E \neq \emptyset\right\}$. Then no cell of level larger than $r$ can be disconnected by $E$. Therefore, we can let $m$ be the level of the "smallest" cells that can be disconnected by elements in $E$ and let $A_{w}$ where $|w|=m$ be one of the "smallest" cells.

Consider the case that $E$ has two elements and let them be $r_{1}=f_{w_{1}}\left(q_{1}\right)$ and $r_{2}=f_{w_{2}}\left(q_{2}\right)$ where $w_{1}, w_{2} \in \Omega^{*}$ and $q_{1}, q_{2} \in \Omega^{*}$. If $w_{1} \neq w_{2}$ then by Property 4, the number of disconnected components in $A \backslash E$ is $\leq(1-1)+$ $(1-1)+1=1$, which is not possible. Therefore, $w_{1}=w_{2}$. Clearly $A_{w_{1}} \subseteq A_{w}$, because otherwise $A_{w}$ cannot be disconnected by $E$. If $A_{w_{1}} \subsetneq A_{w}$ then $A_{w_{1}} \backslash E$ and $A_{w} \backslash A_{w_{1}}$ are both connected. Thus $A_{w}$ is connected, which is not possible. Therefore, $w=w_{1}=w_{2}$.

Next consider the case that $A$ has three elements and let them be $r_{i}=$ $f_{w_{i}}\left(q_{i}\right)$ where $w_{i} \in \Omega^{*}$ and $q_{i} \in Q^{\prime}$ for $i=1,2,3$. Again from [BR], we cannot have the $w_{i}$ 's all distinct. We may assume $w_{1}=w_{2}$. If $w_{1} \neq w_{3}$, and removing $r_{1}$ and $r_{2}$ cannot disconnect $A_{w_{1}}$ into two pieces, then the number of disconnected components in $A \backslash E$ is $\leq(1-1)+(1-1)+1=1$, which is not possible. So either $A_{w_{1}} \backslash\left\{w_{1}, w_{2}\right\}$ is disconnected or $w_{1}=w_{2}=w_{3}$. If $w_{1} \neq w_{3}$, then since $A_{w} \backslash\left\{r_{3}\right\}$ is connected, $A_{w_{1}} \backslash E$ must be disconnected 
and $A_{w_{1}} \subsetneq A_{w}$, which contradicts the definition of $A_{w}$. Hence, $w=w_{1}=$ $w_{2}=w_{3}$.

The graph in Figure 5 shows all $f_{w}\left(Q^{\prime}\right)=\left\{q_{i} \mid i=1, \ldots, 6\right\}$ and cells of level 1 of $A_{w}$.

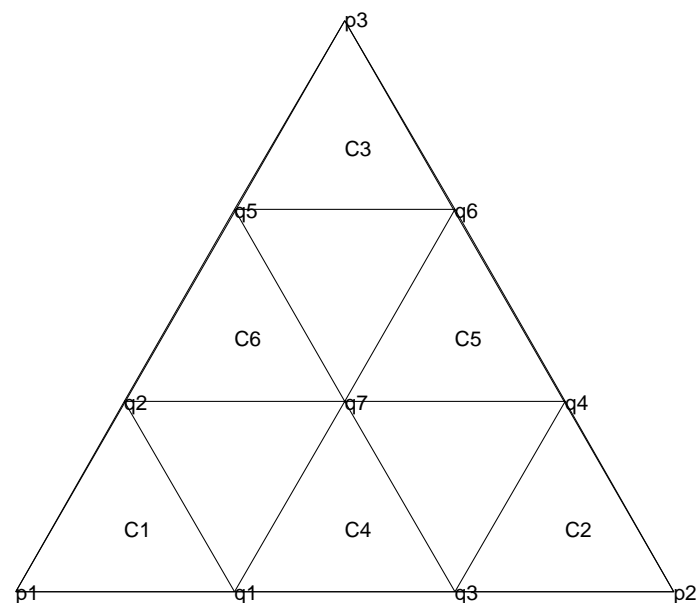

Fig. 5

We see that there are three collections of cells, $B_{1}=\left\{C_{1}, C_{4}, C_{6}\right\}, B_{2}=$ $\left\{C_{2}, C_{4}, C_{5}\right\}, B_{3}=\left\{C_{3}, C_{5}, C_{6}\right\}$, with the property that the three cells in each collection intersect each other at boundary points. Let $D_{i}=\bigcup_{C \in B_{i}} C$ for $i=1,2,3$. If all $D_{i} \backslash E$ are connected then since $\left(D_{i} \backslash E\right) \cap\left(D_{j} \backslash E\right) \neq \emptyset$ for all $i, j \in\{1,2,3\}$, it follows that $A_{w} \backslash E=\left(D_{1} \cup D_{2} \cup D_{3}\right) \backslash E$ is connected, which contradicts our assumption.

Therefore, at least one pair of points $\{a, b\}$ in $\left\{\left\{q_{2 i-1}, q_{2 i}\right\} \mid i=1,2,3\right\}$ $\cup\left\{\left\{q_{7}, q_{i}\right\} \mid i=1,2,3,4,5,6\right\}$ must be in $E$. If there exist $c \in E$ in the interior of any one of the six cells, $A$ can be disconnected using $a$ and $b$ only. Hence, in the closure of each disconnected component we can find either a cell with both $a$ and $b$ as boundary points, or two cells that intersect at a boundary point with the property that one cell has $a$ as boundary point and the other has $b$ as boundary point. This is summarized in the following lemma.

Lemma 3.1. Let $E$ be a set of two or three points that can cut $A$ into two pieces but no proper subset of $E$ can do so. Let $D_{1}^{\prime}$ and $D_{2}^{\prime}$ be the two disconnected pieces and let $D_{i}=D_{i}^{\prime} \cup E$ for $i=1,2$. Then one of $D_{1}$ and $D_{2}$ has a subset that is homeomorphic to $S G_{3}$ and contains two points in $E$ as boundary points, while the other has two subsets $H_{1}$ and $H_{2}$ that are homeomorphic to $S_{3}$, with a unique intersection point that is a boundary point of both of them, and each contains exactly one point in $E$ as a boundary point. 
Lemma 3.2. A cannot be disconnected into three pieces by three points.

Proof. Assume that there exists a set of three points $E$ such that $A \backslash E$ is disconnected into three pieces. We know that the three points must be of the form $F_{w}(q)$. For all $v \in \Omega^{*}$, we let $E_{v}=E \cap F_{v}\left(Q^{\prime}\right)$ and $V=$ $\left\{v \in \Omega^{*} \mid E_{v} \neq \emptyset\right\}$ and $k_{v}$ be the number of components of $A_{v} \backslash E_{v}$. From Property 4 , we know that $3-1 \leq \sum_{v \in V}\left(k_{v}-1\right)$. If $\operatorname{card}(V) \geq 2$ then we would have $3-1 \leq(2-1)+(1-1)$ or $3-1 \leq(1-1)+(1-1)+(1-1)$, a contradiction. Therefore, $\operatorname{card}(V)=1$. Let $\{w\}=V$. We can observe directly from the $G_{1}$ graph of $A_{w}$ that $A_{w}$ cannot be disconnected into three pieces by three points. Therefore, $A$ cannot be disconnected into three pieces by three points.

Definition 3.3. Any subset $S$ of $\mathbb{R}^{n}$ is said to be a copy of $S G_{3}$ if it is homeomorphic to $A$. Let $h_{1}$ and $h_{2}$ be two homeomorphisms from $A$ onto $S$. Then $\left(h_{1}\right)^{-1} \circ h_{2}$ is a homeomorphism from $A$ onto $A$. Therefore, we must have $h_{1}(V)=h_{2}(V)$, and we call elements in $h_{1}(V)$ boundary points of $S$. Moreover, we let $\operatorname{Int}(S)=S \backslash h_{1}(V)$ be the interior of $S$.

$\mathcal{F}$ is an $S G_{3}$ fractafold if it is made up of copies $S_{1}, \ldots, S_{m} \subset \mathbb{R}^{n}$ of $S G_{3}$ intersecting only at boundary points. We call the set $\left\{S_{1}, \ldots, S_{m}\right\}$ a decomposition of $\mathcal{F}$. Clearly, $\mathcal{F}$ can have more than one decomposition. We call $D=\left\{S_{1}, \ldots, S_{m}\right\}$ a minimal decomposition if $D$ does not have a subset $D^{\prime}$ with the following properties:

(A1) $\operatorname{card}\left(D^{\prime}\right) \geq 2$,

(A2) $D^{*}=\bigcup_{S \in D^{\prime}} S$ is a copy of $S G_{3}$, and no interior point of $D^{*}$ belongs to any $S \in D \backslash D^{\prime}$. This implies that if $B$ is a subset of $\bigcup_{S \in D \backslash D^{\prime}} S$ and is a copy of $S G_{3}$ then it either does not intersect $D^{*}$ or intersects $D^{*}$ only at the boundary point of both of them.

Lemma 3.4. Given any subset $F$ of $\mathcal{F}$ that is a copy of $S G_{3}$ and a decomposition $D=\left\{S_{1}, \ldots, S_{m}\right\}$ of $\mathcal{F}$. If $F \cap \operatorname{Int}(S) \neq \emptyset$ for some $S \in D$ and $F$ is not a subset of $S$, then $S$ is a subset of $F$.

Proof. Let $V_{S}=\{a, b, c\}$ be the set of boundary points of $S$. Then $\operatorname{card}\left(F \cap V_{S}\right) \geq 2$. Otherwise, $F$ can be disconnected using only one point. Clearly, elements in $F \cap V_{S}$ can cut $F$ into two pieces $D_{1}^{\prime}$ and $D_{2}^{\prime}$. There are two possible cases:

CASE 1: Two points $a^{*}$ and $b^{*}$ in $F \cap V_{S}$ can cut $F$ into two pieces.

CASE 2: $\operatorname{card}\left(F \cap V_{S}\right)=3$ and all the three points in $F \cap V_{S}$ are required to cut $F$ into two pieces.

In Case 2, it is clear that one of $D_{1}^{\prime}$ and $D_{2}^{\prime}$ must be a subset of $S$. In Case 1 , one of $D_{1}^{\prime}$ and $D_{2}^{\prime}$ must intersect the interior of $S$. If it is not a subset of $S$ then it can be cut into two pieces by the point in $V_{S} \backslash\left\{a^{*}, b^{*}\right\}$. 
This shows that $F$ can be cut into three pieces using three points, which contradicts Lemma 3.2.

From Lemma 3.1, there exist two points $a^{\prime}$ and $b^{\prime}$ in $V_{S}$ such that there exist two subsets $C_{a}$ and $C_{b}$ of $S \cap F$ with the following properties:

(P1) Both $C_{a}$ and $C_{b}$ are homeomorphic to $S G_{3}$. By Property 2, they are cells of both $S$ and $F$.

(P2) $a^{\prime}$ is a boundary point of $C_{a}$ while $b^{\prime}$ is a boundary point of $C_{b}$.

(P3) $C_{a} \cap C_{b} \neq \emptyset$.

Therefore, $C_{a}=C_{b}=S$ and $S \subseteq F$.

TheOrem 3.5. Any fractafold $\mathcal{F}$ of $S_{3}$ has a unique minimal decomposition.

Proof. Given any decomposition $D$ of $\mathcal{F}$, we can form a minimal decomposition by continuously replacing subsets $D^{\prime}$ of $D$ with property (A1) and (A2) by one copy of $S G_{3}$. The process must terminate because the number of elements in $D$ is finite.

Now, let $D_{1}$ and $D_{2}$ be two minimal decompositions of $\mathcal{F}$. If $D_{1} \neq D_{2}$ then without loss of generality there exists a set $S_{1}$ in $D_{1}$ such that $S_{1} \notin D_{2}$. Then there exists $S_{2}$ in $D_{2}$ such that either $S_{1} \subsetneq S_{2}$ or $S_{1} \supsetneq S_{2}$ by Lemma 3.4. If $S_{1} \subsetneq S_{2}$, then let

$$
A=\left\{S \in D_{1} \mid S \cap \operatorname{Int}\left(S_{2}\right) \neq \emptyset\right\} .
$$

By Lemma 3.4, we have $A=\left\{S \in D_{1} \mid S \subsetneq S_{2}\right\}$ and $S_{2}=\bigcup_{S \in A} S$. We will show that $A \subset D_{1}$ satisfies conditions (A1) and (A2), which contradicts the fact that $D_{1}$ is a minimal decomposition. Condition (A1) is trivial. If there exist $S^{\prime} \in D_{1} \backslash A$ such that $S^{\prime} \cap \operatorname{Int}\left(S_{2}\right) \neq \emptyset$, since $S^{\prime}$ is $S G_{3}$, we have $S^{\prime} \subseteq S_{2}$ or $S^{\prime} \supseteq S_{2}$ by Lemma 3.4, which is not possible. This establishes condition (A2).

The case of $S_{2} \subsetneq S_{1}$ is similar.

THEOREM 3.6. Let $\mathcal{F}$ be a fractafold based on $S G_{3}$. Any homeomorphism of $\mathcal{F}$ onto $\mathcal{F}$ must be a permutation of the elements in the minimal decomposition $D^{*}$.

Proof. $h\left(D^{*}\right)=\left\{h(S) \mid S \in D^{*}\right\}$ is also a minimal decomposition. By Theorem 3.5 , we have $h\left(D^{*}\right)=D^{*}$.

Acknowledgments. Y. Y. Chan wishes to thank the Mathematics Department of the Chinese University of Hong Kong for travel support to Cornell University.

Research of R. S. Strichartz was supported in part by the National Science Foundation, Grant DMS-0652440. 


\section{References}

[BK] C. Bandt and K. Keller, Self-similar sets 2. A simple approach to the topological structure of fractals, Math. Nachr. 154 (1991), 27-39.

[BR] C. Bandt and T. Retta, Topological spaces admitting a unique fractal structure, Fund. Math. 141 (1992), 257-268.

[Ki] J. Kigami, Analysis on Fractals, Cambridge Univ. Press, Cambridge, 2001.

[MSW] D. Mumford, C. Series and D. Wright, Indra's Pearls. The Vision of Felix Klein, Cambridge Univ. Press, Cambridge, 2002.

[RS] H.-J. Ruan and R. Strichartz, Covering maps and periodic functions on higher dimensional Sierpiński gaskets, Canad. J. Math. 61 (2009), 1151-1181.

[S1] R. Strichartz, Fractafolds based on the Sierpinski gasket and their spectra, Trans. Amer. Math. Soc. 335 (2003), 4019-4043.

[S2] -, Differential Equations on Fractals: a Tutorial, Princeton Univ. Press, Princeton, 2006.

[S3] - Periodic and almost periodic functions on infinite Sierpiński gaskets, Canad. J. Math. 61 (2009), 1182-1200.

Ying Ying Chan

Mathematics Department

Chinese University of Hong Kong

Shatin, Hong Kong

E-mail: yingyingsharon@gmail.com
Robert S. Strichartz

Mathematics Department Malott Hall

Cornell University

Ithaca, NY 14853, U.S.A.

E-mail: str@math.cornell.edu

Received 11 June 2009;

in revised form 29 April 2010 